Review

\title{
Nanoparticle-Liquid Crystalline Elastomer Composites
}

\author{
Yan Ji ${ }^{1,2}$, Jean E. Marshall ${ }^{1}$ and Eugene M. Terentjev ${ }^{1, *}$
}

1 Cavendish Laboratory, University of Cambridge, JJ Thomson Avenue, Cambridge CB3 OHE, UK;

E-Mails: yj230@cam.ac.uk (Y.J.); jec60@cam.ac.uk (J.E.M.)

2 Department of Chemistry, Tsinghua University, Beijing 100084, China

* Author to whom correspondence should be addressed; E-Mail: emt1000@cam.ac.uk;

Tel.: +44-1223-337-003; Fax: +44-1223-337-000.

Received: 30 November 2011; in revised form: 10 January 2012 / Accepted: 14 January 2012 /

Published: 30 January 2012

\begin{abstract}
Liquid crystalline elastomers (LCEs) exhibit a number of remarkable physical effects, including a uniquely high-stroke reversible mechanical actuation triggered by external stimuli. Fundamentally, all such stimuli affect the degree of liquid crystalline order in the polymer chains cross-linked into an elastic network. Heat and the resulting thermal actuation act by promoting entropic disorder, as does the addition of solvents. Photo-isomerization is another mechanism of actuation, reducing the orientational order by diminishing the fraction of active rod-like mesogenic units, mostly studied for azobenzene derivatives incorporated into the LCE composition. Embedding nanoparticles provides a new, promising strategy to add functionality to LCEs and ultimately enhance their performance as sensors and actuators. The motivation for the combination of nanoparticles with LCEs is to provide better-controlled actuation stimuli, such as electric and magnetic fields, and broad-spectrum light, by selecting and configuring the appropriate nanoparticles in the LCE matrix. Here we give an overview of recent advances in this area with a focus on preparation, physical properties and actuation performance of the resultant nanocomposites.
\end{abstract}

Keywords: liquid crystal; elastomer; actuation; nanoparticles; nanotubes

\section{Introduction}

Smart materials, which are capable of converting external stimuli into mechanical responses, have long been recognized as an exciting and high-impact research area with widespread applications [1,2]. 
Several different types of responsive materials have been developed so far, with varying mechanical properties and response mechanisms. Of these, liquid crystal elastomers (LCEs) are a particularly useful and interesting class of materials that combine softness, elasticity, durability, light weight, high force density, and notable mechanical strength with dramatic reversible actuation [3]. They have been studied as valuable candidates for artificial muscles for some time, and they also exhibit interesting potential as motors [4,5], contact lenses [6], microgrippers in microsystems [7], valves for microfludics [8] and tunable lasing media [9-13].

In simple terms, LCEs are weakly cross-linked elastic polymer networks that incorporate rigid, anisotropic units (termed mesogens) bonded to the polymer chains. The mesogens can exhibit spontaneous orientational ordering, of the same type that is observed in ordinary low molar mass liquid crystals. However, since the mesogens are chemically bound to the cross-linked polymer network, any change in their orientation will be coupled to changes in the mechanical properties of the bulk material. If there is an overall preferred direction for the mesogens (termed the liquid crystal director) and this direction is the same throughout the material, then this network can be termed a mono-domain LCE (in contrast to a poly-domain LCE, in which each domain inside the material has a different director, see [14-18] for a summary and details of poly-domain to mono-domain transition in LCE). As the mesogens undergo a phase transition from the liquid crystalline state to the isotropic state, mono-domain LCEs exhibit a significant change of their macroscopic shape due to the fact that motion of the polymer network is coupled to that of the mesogens bound to the polymer. The transition between the ordered and disordered states is dictated by the thermodynamic equilibrium; hence the shape change is fully reversible. After this phenomenon was originally predicted by De Gennes [19,20] and experimentally confirmed by Finkelmann [21], theoretical and experimental studies in this area proliferated, driven both by scientific interest in this new functional soft matter (which combines both rubber-like elasticity and anisotropic liquid crystalline ordering), and by technological interest in the potential applications of such a material. Various types of LCEs have been developed. Uniaxial deformations of well over $300 \%$ (in the direction of the LC director) during the phase change have been demonstrated [22]. LCEs also demonstrate other interesting physical features (such as soft elasticity, mechanical and electric instabilities) but it is this spontaneous reversible deformation - giving them a potentially large actuation stroke - that currently makes them stand out from other soft actuators.

The incorporation of nanoparticles into LCEs has been of considerable recent interest, with the aim of achieving a faster response speed and better control over the actuation. In most LCE systems, the large deformations seen can be achieved by heating the material, in order to trigger the LC change into the isotropic phase. However, the low thermal conductivity of polymeric LCEs prevents quick delivery of heat to all mesogens in the material, thus impeding the LC-isotropic phase transition-so that for thick and large LCE films, or indeed other shapes, the response time is an essential drawback for many applications. For practical applications, other drawbacks of direct heating are the difficulty of creating a heat supply that would change the surrounding temperature of LCEs at a very high speed, and the difficulty of creating a heat stimulus that would be entirely localized around the LCE component (so that adjacent actuating features could be stimulated independently). Even if such a heating mechanism were available, it would be difficult to incorporate it into a practical device. For easy manipulation of the actuating component, other stimuli (e.g., light, electric and magnetic fields) are far more appealing 
and it is with the goal of sensitizing LCEs to these stimuli in mind that researchers turn to the idea of incorporating other components into the material.

Since the underlying mechanism of actuation is the switching between the LC (in most cases - nematic) phase and the isotropic phase, in principle, any external source that could activate such a phase transition can be used to trigger the actuation. One strategy for sensitizing LCEs to an external energy source is to modify them with stimuli-responsive organic functional groups, either by covalently attaching these to the polymeric chains $[23,24]$ or by physically adsorbing them into swelling LCEs [25]. Several photo-responsive LCEs have been developed by introducing photochromic groups into the LCEs [26,27]. The majority of these photochromic molecules are azobenzene derivatives, although stilbene derivatives are also studied. Upon photo-irradiation with a suitable (UV) light source, the azobenzene group undergoes a trans-cis isomerization; since the trans form is a rod-like LC mesogen, while the cis form shows no mesogenic behavior, this process can cause a phase change in the LC ordered material and hence a deformation in a mono-domain material. Using polarized light, movement can even be induced in un-aligned poly-domain LCEs, thus considerably simplifying the preparation process for these materials [28]. The detailed mechanism has been investigated [29]. Recently, photoresponsive main-chain LCEs with azobenzene moieties have been developed and more interesting work has been reported [30-33]. However, these materials do have inherent limitations, connected to the penetration depth of light within the material; if light only penetrates the surface layer of the LCEs, then only the surface chromophores will receive the light stimulus and the material will bend rather than contract. To achieve contraction of the LCE, the film needs to be thin and the material needs to be exposed to light over a long time period (of the order of minutes to hours) in order to accumulate enough light energy [23,34]. An alternative LCE modification involves the incorporation of ferroelectric mesogens (having a high dielectric anisotropy) in order to sensitize the LCEs to an electric field [35-37]. These mesogens in side-chain LCEs can change their alignment in response to an external electric field, resulting in an electromechanical effect with response times that can be of the order of $10 \mathrm{~ms}$. Bent core LCEs have been developed to produce giant flexoelectricity for microscale parasitic power generation [38,39]. Toward this end, main-chain ferroelectric smectic LCEs were synthesized, with analysis of shear deformation and ferroelectricity [40]. However, strong electric fields are necessary to produce actuation in ferroelectric LCEs, and the amplitudes of the resulting mechanical contractions are quite small [35].

Resistive joule heating provides an alternative approach to the control of LCEs using electrical stimuli. The most straightforward method is to embed wires into the LCE matrix [41]. When an electrical field is applied, as an ohmic resistance, the wires generate heat. The increased temperature causes the mesogens to undergo a phase change and contract, as in the case of direct heating. However, the wires are stiff and can thus restrict the LCEs from undergoing a large deformation. This method also faces critical heat transfer problems. A thin layer of conducting carbon on the LCE surface has the same shortcoming, with loss of mechanical response [42-46].

One obvious possibility for sensitizing LCE materials to remote stimuli such as light (as opposed to outside heating) is to add functional nanoparticles to the material [47]. Nanoparticles have engaged intense scientific and industrial interest over a wide range of areas, from biomedicine to electrical components; their small size (less than $100 \mathrm{~nm}$ in at least one dimension) means that a relatively large fraction of their constituent atoms are surface atoms, a characteristic which can give them unusual 
physical and chemical properties compared to the corresponding bulk materials. The recent expansion in nanotechnology research means that an overwhelming variety of nanoparticles are now available; incorporating these into polymer materials allows the development of new composites with novel optical, electrical or mechanical properties. We are interested in composites created by combining nanoparticles with LCEs, in such a way that the nanoparticle response to light or electric/magnetic fields can be transferred to the LCE matrix, triggering mechanical motion. The presence of nanoparticles in the LCEs can also improve the materials' response times, as well as making the material sensitive to different types of stimuli.

Nanoparticles can be processed into a thin layer on the LCE surface or distributed homogenously in the bulk LCEs. Some systematic work has been done with carbon reprocessed LCEs, where the pre-formed mono-domain LCEs were swollen in a solution containing carbon nanoparticles; evaporating the solvent from this solution leaves a thin conducting carbon layer on the surface of the LCE. Such a layer allows resistive heating in an electric field, resulting in electrical actuation with little loss of mechanical response. The mechanism, processing, electro-thermo-mechanical properties, material degradation and accompanied piezoresistivity effect of carbon reprocessed LCEs have been extensively reviewed previously [44-46], and will therefore not be included here. Herein, we summarize recent advances in LCE nanocomposites, with reference to representative original publications including our own work. We will begin by discussing the selection of nanoparticles and LCEs available, and will subsequently describe the resulting thermal properties and actuation of the resulting nanocomposite materials. We restrict ourselves to systems where nanoparticles are incorporated into mono-domain LCEs with emphasis given to the problems encountered in nanocomposite preparation. The review concludes with some relevant challenges and perspectives of this field to stimulate further discussions on design, fabrication and performance, and research interest in these nanocomposites.

\section{Selection of Nanoparticles and LCEs}

With the aim of promoting fast response times and sensitivity to multiple stimuli, the nanoparticles selected for LCE composites must possess special optical, electrical or magnetic properties. They could be broadly divided into two categories: carbon and non-carbon nanoparticles.

Carbon-based nanoparticles are usually commercially available. Carbon nanoparticles, carbon nanohorns, and carbon nanotubes have been described as electric and/or light induced nanoscale heating sources for fast actuation of LCEs. Carbon nanotubes particularly have been shown to effectively absorb light and efficiently convert this to heat. Deformable elastomers with embedded carbon nanoparticles as conducting layers have been summarized in [48]. Carbon nanotubes (CNTs) are hollow cylinders that essentially consist of rolled-up graphene sheets; these typically have a diameter of the order of nanometres and length of the order of microns. Single-walled carbon nanotubes (SWNTs) are made of a single rolled sheet of graphene. Multi-walled carbon nanotubes (MWNTs) contain a number of concentric cylinders. Compared with other nanoparticles, they have extremely high surface-to-volume ratio and rare anisotropic thermal, mechanical, and electronic properties [49]. They can be metallic, or semiconducting in the case of some SWNTs. Their electric current carrying capacity is even higher than that of copper. Meanwhile, they display outstanding chemical and thermal stability. Several successful attempts have been reported on incorporating CNTs into low molar mass liquid crystalline 
fluids [50,51], with a resulting change in electro-optical response. By introducing CNTs into LCEs, it has been demonstrated that the composite material is sensitized to both light and electrical fields [52,53]. Since CNTs also respond to magnetic fields, the CNT-LCE composite could potentially also be manipulated by a magnet. SWNTs or MWNTs could be incorporated into the LCE matrix in order to deliver local heat to the LC mesogens. However, the actuation of LCE-CNT composites is not totally reliant on the thermal effect after light/electric activation; this has the advantage that in both electric and light-driven actuation, the temperature can be far below the nematic-isotropic temperature and still produce measurable deformation.

Non-carbon inclusions include electrically active and magnetically active nanoparticles. Most of these are not commercially available and must therefore be synthesized in the lab. Domenici et al. deposited a layer of $\mathrm{MoO}_{3-x}$ nanowires onto the LCE surface, prepared by special treatment of $\mathrm{Mo}_{6} \mathrm{~S}_{2} \mathrm{I}_{8}$ nanowires [54]. These nanowires consisted of $\mathrm{MoO}_{3}$ and $\mathrm{Mo}_{5} \mathrm{O}_{14}$ crystals. The length of these nanowires is similar to that of carbon nanotubes and can be several millimeters, but they have larger diameters (up to $500 \mathrm{~nm}$ ). They are porous, and have triangular thin flakes jutting out of the surface, perpendicular to the nanowire axis. These nanowires are electrically conductive, and are therefore interesting for their possible effect on the LCE actuation properties.

Perovskite-based ferroelectric $\mathrm{PbTiO}_{3}$ nanoparticles have also been used to form composites with LCEs [55]. The $\mathrm{PbTiO}_{3}$ nanoparticles were synthesized by sequential precipitation of $\mathrm{TiCl}_{4}$ and $\mathrm{Ph}\left(\mathrm{NO}_{3}\right)$ followed by calcination. The resulting nanoparticles were somewhat elongated and polydisperse, with a size distribution in the $70-800 \mathrm{~nm}$ range. The authors hoped that the high ferroelectricity of these particles could be used to trigger actuation in LCE composite materials.

Magnetically active LCEs were reported by Kaiser et al. and Winkler et al., using superparamagnetic $\mathrm{Fe}_{3} \mathrm{O}_{4}$ nanoparticles [56,57]. The $\mathrm{Fe}_{3} \mathrm{O}_{4}$ nanoparticles were prepared by alkaline hydrolysis of a mixture of ferrous and ferric chloride. These nanoparticles were stabilized in toluene with $\mathrm{N}$-oleoylsarcosine; this could then be used as the liquid medium for the preparation of LCEs. The $\mathrm{F}_{3} \mathrm{O}_{4}$ nanoparticles had an average diameter of $12 \mathrm{~nm}$. The authors intended to use the resulting composites as antennae for electromagnetic energy dissipation; under an alternating magnetic field heat was also produced due to hysteresis, triggering the nematic-isotropic transition.

A typical LCE usually consists of three basic components: the mesogens, the polymer backbone and the crosslinkers. The mesogens are either attached to the polymeric backbone as side groups (forming "side-chain LCEs") or are included as portions of the polymer backbone (resulting in "main-chain LCEs"). These are the key elements needed for formation of the liquid crystalline phase. The crosslinkers covalently connect different polymer chains to build a network. As in the case of small-molecule LCs, different chemical structures enable them to form different liquid crystalline phases including nematic, smectic and cholesteric phases. In all of these phases the mesogens have some orientational order (i.e., a preferred direction), but the phases differ in the amount and the symmetry of positional order present. So far, the published literature on composites containing nanoparticles and mono-domain LCEs has focused on nematic side-chain LCEs. Most studies chose LCEs based on polysiloxane, especially the one based on the hydrosilylation of polyhydrogenmethylsilxoane with 4-methoxyphenyl-4-buteneoxy benzoate (MBB) as mesogen and 1,4-di-11-undeceneoxy benzene (11UB) as crosslinker, see Figure 1(a); this system has been originally developed by Finkelmann [21] and extensively characterized over the years. Parameters such as crosslinker concentration, reaction 
temperature, catalyst, and solvent vary slightly between different studies. The resulting LCEs exhibit an isotropic-nematic transition temperature around $80-90{ }^{\circ} \mathrm{C}$.

Figure 1. Chemical structure of LCE starting materials: (a) polysiloxane-based LCE, and (b) LCE with acrylate backbone.

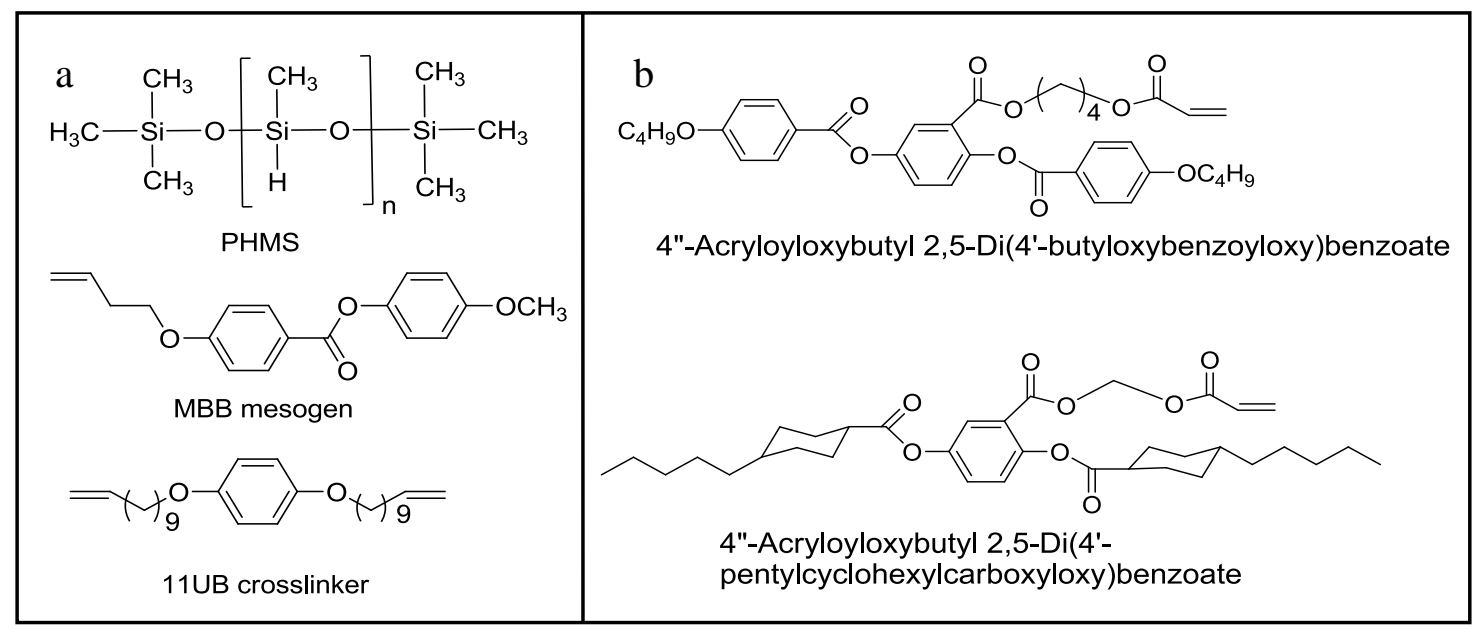

An alternative side-chain nematic LCE can be formed from mesogens containing acrylate groups [58-60]. The acrylate groups can be polymerized under UV light in the presence of a suitable photo-initiator, or can undergo radical polymerization. Various acrylate-containing mesogens have been used for this purpose, but these suffer from the disadvantage that the resulting carbon backbone chain is less flexible than that of the siloxane polymers; the $T_{g}$ of the acrylate polymers is therefore often rather high, reducing the ability of the polymer to actuate (because the motion of the LC mesogens is restricted while the polymer is in a glassy state). Strictly speaking, they are glassy LC networks at room temperature. The work of Thomsen et al. demonstrated a solution to this problem; by co-polymerising a mesogenic acrylate with a non-mesogenic acrylate, the glass transition temperature was successfully reduced, Figure 1(b) [61]. Yang et al. showed that suitably surface-modified SWNTs have good compatibility with this type of polymer system, and that adding SWNTs to the polymer increases the sensitivity of the material to light [62]. The resulting LCE has a nematic-isotropic transition at about $52.5^{\circ} \mathrm{C}$ upon heating and $39.8^{\circ} \mathrm{C}$ during cooling.

\section{Processing}

A major breakthrough was achieved in the field of LCEs when the group of Finkelmann successfully synthesised mono-domain LCEs using a two-step cross-linking method [21]. Firstly, the starting materials (siloxane polymer, mesogen, cross-linker and catalyst) reacted to form a partially cross-linked gel-like network. This gel-like network was then stretched mechnically, to induce a uniform orientation of the LC mesogens; a second cross-linking step was then carried out in order to fix this orientation. Using this procedure, the mesogens are aligned macroscopically. If no uniaxial stretching is applied, the film will become poly-domain on final cross-linking, with the nematic director of each domain being randomly arranged with a characteristic correlation length scale of the order of microns. The two-step crosslinking method is the most widely adopted procedure for the preparation of nanoparticle-enriched LCEs. The nanoparticles may be simply mixed with the other starting materials 
before the first cross-linking step, and will consequently remain embedded in the polymer as it is fully cross-linked. In order to use this method, some reaction parameters may need optimization in the presence of nanoparticles. For example, in some circumstances, the presence of carbon nanoparticles inhibits the formation of an elastomer network with liquid crystalline properties. We found that CNTs can impede the polymerization process, and that a catalysis system optimized for pure LCEs must be adjusted when CNTs are included; in our experience, Karstedt's catalyst (Platinum(0)-1,3-divinyl1,1,3,3-tetramethyldisiloxane) is more effective than the often-used $\mathrm{Pt}(\mathrm{COD}) \mathrm{Cl}_{2}$ when $\mathrm{CNT}$ s are part of the first gelation step.

For this first step, a common practice (based on work in the group of H. Finkelmann) is to use a centrifuge fitted with specially designed reactor to obtain a partially cross-linked gel-like film with uniform thickness. The reaction mixture with the nanoparticles is deposited in the reactor, which has a PTFE film lining its inner wall. The reactor is placed in a centrifuge at a fixed centrifugation speed and temperature. However, unmodified nanoparticles easily precipitate from the reaction mixture and form a layer on the surface of the film. This was used by Domenici et al. to prepare nanoparticle layers on the top of LCEs $[54,55]$. SWNTs were also observed to precipitate out of the reaction mixture, and to aggregate severely during centrifugation. To avoid the formation of such layers, the first cross-linking can be performed by molded casting instead of centrifugation [63]. Yang et al. [62] also used the casting method to prepare homogeneous SWNT-LCE nanocomposite.

It is energetically favorable for nanoparticles to aggregate, which reduces their surface energy. This tendency is a critical challenge in the preparation of mono-domain LCE nanocomposites. It makes mixing the nanoparticles with the reaction mixture difficult, and has a profound effect of the relevant properties of the final nanocomposite. If there is too much aggregation and the nanoparticles are not compatible with LCEs in terms of their elastic modulus, then poor stress transfer will occur, resulting in interfacial slippage as reported in other polymer nanocomposites [64] and large cracks are observed in LCEs with conducting layers [44]. Moreover, the presence of microscopic aggregates could badly disrupt the underlying liquid crystalline order. Poor dispersion of nanoparticles in the polymer matrix can result in inferior mechanical properties and deficient actuation performance. The problem is especially severe for carbon nanotubes. Pristine CNTs have an overpowering tendency to agglomerate, rapidly forming bundles because of their high aspect ratio and the strong van der Waals attractions between tubes. Despite promising applications envisaged for CNTs across a wide range of disciplines, poor solubility is one of the most fundamental challenges encountered in attempts to make use of CNTs' extraordinary properties. Even after being subjected to ultrasound for several hours, they can still remain in the form of clusters, as demonstrated in Figure 2(a). To prepare well-aligned mono-domain LCEs in the presence of CNTs proved even more difficult, especially with a high loading of CNTs.

The incorporation of nanoparticles into LCEs is only worthwhile if the nanoparticles result in a quick response time/alternative actuation pathway, while preserving the liquid crystalline properties and thermomechanical capabilities of the LCE. The use of carbon reprocessed LCEs (made by swelling the neat LCEs in solutions containing carbon nanoparticles) goes some way towards addressing this problem; since the nanoparticles are not in the inner part of the LCEs, the polymer keeps its usual LC phase and thermomechanical actuation [44]. Conducting layers formed by $\mathrm{MoO}_{3-x}$ and $\mathrm{PbTiO}_{3}$ particles separating from the reaction mixture during centrifugation are also assumed to behave like an outer carbon layer [54,55]. However, as in the case of embedded wires [41], when the conducting 
layers are formed on the LCE, temperature gradients and mechanical incompatibility result in quick degradation. Cracks appeared after several actuation cycles. Therefore, quite a lot of effort is dedicated to effective dispersion of nanoparticles in bulk LCEs.

Figure 2. (a) Clustered multi-walled carbon nanotubes (MWNTs) after sonication in pure petroleum ether; (b) poly(p-phenyleneethynylene) used for single-walled carbon nanotube (SWNT) dispersion; (c) chemical structure of PyMC; (d) the morphology of the well-separated carbon nanotubes (CNTs) after the PyMC was washed away.
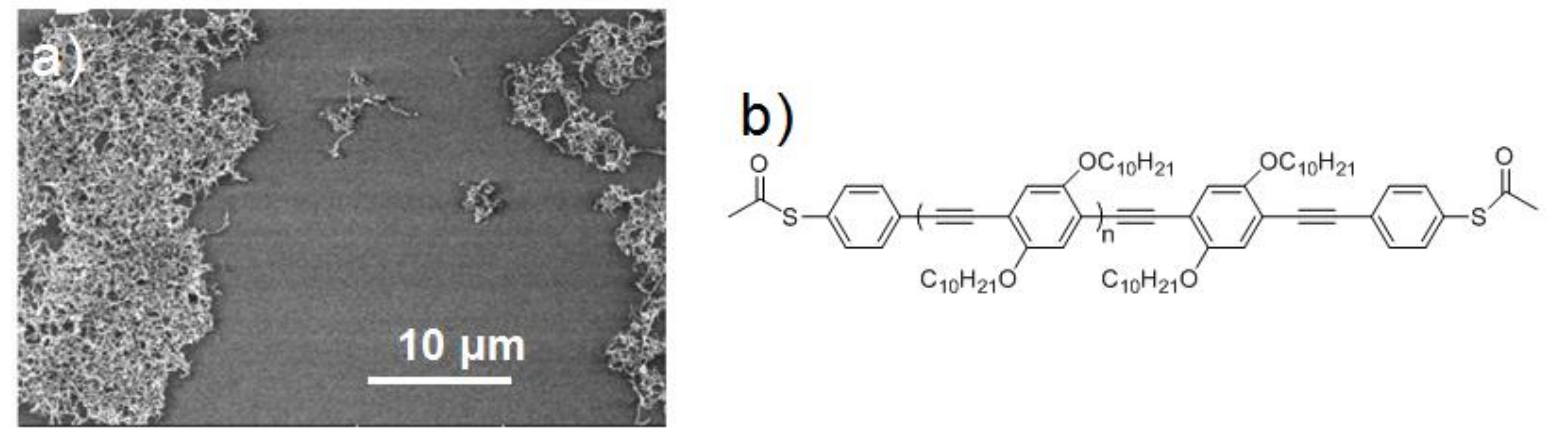

c)
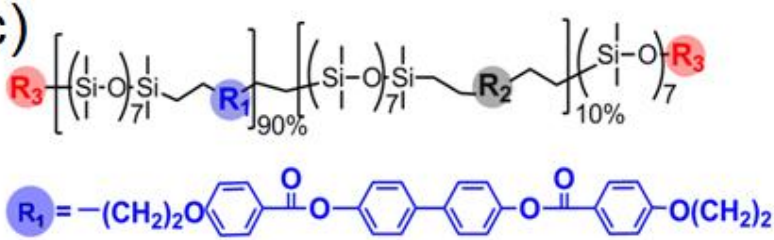

$\mathrm{R}_{2}=-\left(\mathrm{CH}_{2}\right)_{9} \mathrm{O}-\left\langle-\mathrm{N}=\mathrm{N}-\longrightarrow-\mathrm{O}\left(\mathrm{CH}_{2}\right)_{9}\right.$
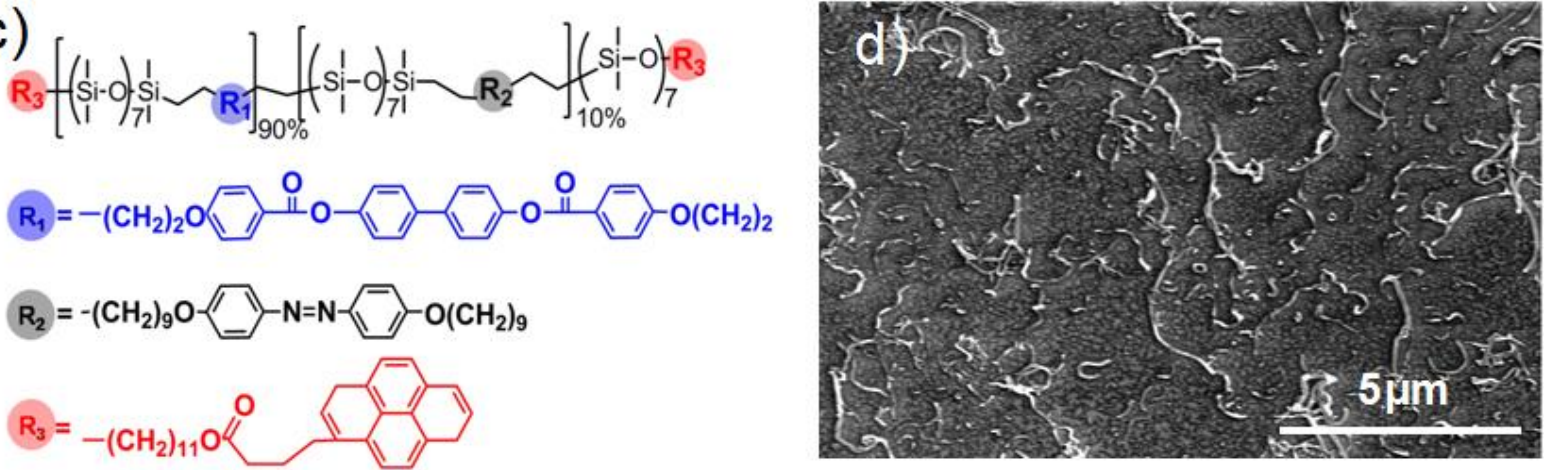

Recognizing that dispersion plays a key role in the preparation of the LCE nanocomposite with a view to maintaining the liquid crystalline ordering, various strategies have been explored in order to homogenously disperse nanoparticles in the matrix. The first approach involves improvement of the mixing method. Both mechanical shearing and ultrasonication have been employed in order to disperse nanoparticles in solution and form a homogenous reaction mixture containing both CNTs and the other components of LCEs. Early experiments involved adding CNTs into the reaction mixture directly, followed by the application of mechanical shear for several hours in order to homogenize the mixture before the addition of catalyst [65].

This method was later modified, such that the CNTs were first dispersed in toluene. The suspension was treated with alternating high-power sonication and mechanical stirring at 2,000 rpm for at least $24 \mathrm{~h}$ [53]. The CNT-toluene suspension was then transferred into the polymerization reaction mixture and stirred for a further $4-6 \mathrm{~h}$ before the addition of the catalyst. Even with this modified method, the maximum concentration of CNTs homogeneously dispersed in the LCE is below 0.02 wt\%. Ultrasonication does usually result in better dispersion, but is unsuitable in some cases as was shown in attempts to disperse $\mathrm{PbTiO}_{3}$ nanoparticles. When $\mathrm{PbTiO}_{3}$ was mixed with toluene, mechanical mixing (>30 min) produced solutions which were stable for up to 5-6 h. However, during ultrasonication the 
nanoparticles adhered to the surface of the glass container, rather than being dispersed in the solvent. Nevertheless, the same research group also showed that ultrasonication was an efficient method for the dispersion of $\mathrm{MoO}_{3-x}$ particles. Even when nanoparticles are evenly dispersed in the reaction mixture, this does not guarantee that they will remain so in the LCE nanocomposite during crosslinking and solvent evaporation. For example, Li et. al. ultrasonicated the reaction mixture with SWNTs for 2 min, but aggregated SWNTs are still present in the material as can be seen with the naked eye [63].

The second key component of a strategy for the effective dispersion of nanoparticles in a LCE is surface functionalization of the nanoparticle surface in order to inhibit their aggregation. This may be achieved by coating the nanoparticle surface with a molecule that will reduce the surface tension between the nanoparticles and the LCE matrix, thus increasing their compatibility. Moreover, this kind of functionalization can make it easier to disperse the nanoparticles in solvent before mixing them with LCE prepolymer mixtures. For example, $\mathrm{F}_{3} \mathrm{O}_{4}$ was functionalized with $\mathrm{N}$-oleoylsarcosine before the polymerization step. For CNTs, covalent modification usually causes damage to the nanotubes, leading to deterioration of their electrical and optical properties. Many surface-active molecules have been developed with the intention of de-bundling pristine CNTs in aqueous solutions [66], and organic solvents [67]. Very few of these, however, can be used to help the dispersion of CNTs into LCEs; this is because they have a tendency to disrupt the liquid crystalline order. In the design of a useful dispersant for the introduction of CNTs into an LCE matrix, polymeric dispersants are preferred to small molecules. Small molecule dispersants usually act to separate CNTs using electrostatic forces or micelle formation, while non-ionic polymeric dispersants are capable of following the "wrapping" mechanism [68,69], thus preventing de-bundled CNTs from re-aggregating even after solvent evaporation. By designing the dispersant polymer to be compatible with the liquid crystalline matrix, the LC order in the material can be preserved. Kang et al. found that a rigid, conjugated polymer, poly(p-phenyleneethynylene) (PPE) is excellently capable of non-covalently modifying SWNTs, see Figure 2(b) $[69,70]$. The PPE-SWNT complex allowed the dispersion of CNTs up to a concentration of 0.1-0.2 wt\% in LCEs formed from acrylates, while not impeding their mono-domain alignment. In our group we tackled the problem of CNT aggregation by designing liquid crystalline polymers capable of interacting with the surface of the CNTs [52]. This improved the CNT-LCE compatibility, with little damage to either the CNTs or the liquid crystalline order. The end-capped flexible main-chain liquid crystalline polymer PyMC, Figure 2(c) can effectively assist the dispersion and stabilization of both pristine SWNTs and MWNTs in common organic solvents to high concentrations. The mesogenic groups (R1) in PyMC interact with the CNTs by aromatic stacking. The affinity between the polymer and the CNTs is further improved by the pyrene moieties (R3) attached to the both ends of the main-chain PyMC. Azobenzene groups (R2) were randomly introduced into to suppress crystallization and lower the glass transition of PyMC. As shown in Figure 2(d), the CNTs are individually well-dispersed in chloroform. The excellent dispersion ability, the relative chemical inertness of PyMC and the compatibility of PyMC with siloxane LCEs allowed us to use this PyMC-CNT dispersion as a stock material in the fabrication of mono-domain LCEs with high loading of CNTs (more than $1 \mathrm{wt} \%$ ) with no phase separation and little sacrifice of their thermal mechanical actuation.

The two-step crosslinking preparation of mono-domain LCEs has an additional benefit: alignment of anisotropic nanoparticles. After the partially cross-linked film was carefully removed from the reactor, the LC mesogens were aligned mechanically by applying uniaxial extension in order to 
produce uniform distribution of the local nematic director along the stretching direction. Anisotropic particles embedded in this rubbery elastic medium experience a similar alignment effect; they choose a preferred orientation during the stretching [71]. Even in a rubber such as ordinary PDMS, which contains no LC mesogens, stretching forces embedded CNTs to align [72]. This may not order all of the particles, but at least some are aligned to some extent. This alignment could be enhanced by the presence of LC mesogens. As proved in our experiment, with the same procedure, alignment of CNTs in mono-domain LCEs is better than in isotropic PDMS [52]. As shown in Figure 3, the CNTs in (b) are almost parallel to the stretching direction while in (a) the CNTs protruded out of the fracture plane. Similar coupling between the local mesogen orientation and the nanoparticles has for some time been a topic of study in the field of small liquid crystals, for organizing/aligning nanoparticles such as carbon nanotubes [73]. Therefore, even though $\mathrm{MoO}_{3-x}$ nanowires form a top layer on the LCE films (rather than being evenly distributed throughout the polymer), the nanowires show alignment parallel to the stretching direction. A similar effect was observed in an LCE doped with $\mathrm{PbTiO}_{3}$ nanoparticles. The exploitation of this alignment effect has important implications not only for the performance of LCEs, but also for the application of nanoparticles- especially CNTs, whose unique properties have so far not been fully used to advantage due to the difficulties encountered in attempts to control their specific orientation [74]. CNTs embedded in an LCE matrix were aligned during stretching and this alignment was maintained in the final crosslinked material; this allowed the material to exhibit not only actuation (driven electrically and by infrared (IR) light), but also an anisotropic THz response [52].

Figure 3. Sketch of a plane strip of nematic elastomer with embedded aligned CNTs. The arrows indicate two planes of freeze-fracture, shown in electron microscopy images of $0.02 \%$ CNT sample: (a) in the plane parallel to the nematic director and the nanotube alignment axis, viewed from the top; (b) in the plane perpendicular to this axis, viewed edge-on. The scale bar in both images is $0.5 \mu \mathrm{m}$.
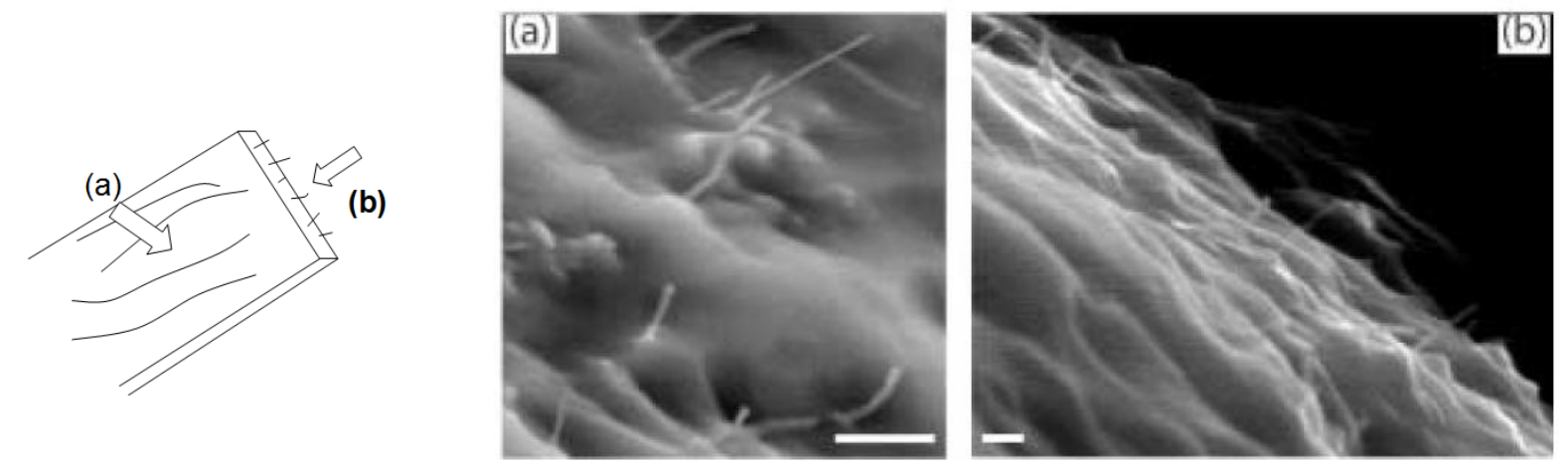

\section{Properties and Actuation of Nanoparticle-Enriched LCEs}

\subsection{Thermal Properties and Thermal Actuation}

It is important to investigate the phase transition properties of the LCE nanocomposite. It is advantageous for the nanoparticles to have as little effect as possible on the macroscopic structure and thermal properties of the material. According to differential scanning calorimetry (DSC) measurements, LCE nanocomposites usually show a thermal behavior similar to that of the "neat" LCE, i.e., the same 
LCE with no added nanoparticles. The presence of a low concentration of nanoparticles results in a slight shift of the nematic-isotropic transition and glass transition temperatures; these tend to be a few degrees lower than is the case in neat LCEs. Introducing $1 \mathrm{wt} \% \mathrm{MoO}_{3-x}$ nanowires in an LCE only decreased the isotropic-nematic temperature by about $1{ }^{\circ} \mathrm{C}$, while the glass transition temperature decreased by less than $4{ }^{\circ} \mathrm{C}$. Meanwhile, the mesophase behavior did not change after several heating and cooling cycles. The transition temperature decrease may in some cases be dependent on the concentration of nanoparticles. In the case of $\mathrm{PbTiO}_{3}$ nanoparticles, the same authors found that the nematic transition point dropped from 74 to 72 to $71{ }^{\circ} \mathrm{C}$ for samples containing $0 \%, 1$ wt $\%$ and $5 \mathrm{wt} \% \mathrm{PbTiO}_{2}$ respectively [55]. The glass transition follows a similar trend: from $-4{ }^{\circ} \mathrm{C}$ to $-6{ }^{\circ} \mathrm{C}$ and $-7.5^{\circ} \mathrm{C}$ for $0 \%, 1 \mathrm{wt} \%$ and $5 \mathrm{wt} \%$ samples. Since the nanoparticles were not homogeneously dispersed in the LCE matrix, and most of the nanoparticles formed a conducting layer on the surface of LCEs, these $T_{n i}$ and $T_{g}$ changes are probably due to the sample preparation instead of the influence of the nanoparticles. In fact, there are studies that show that the transitions are within a particular range and that the variation in the transition temperatures is not correlated to the concentration of nanoparticles. LCEs containing dispersed superparamagnetic nanoparticles have a nematic transition temperature within $69-74{ }^{\circ} \mathrm{C}$, while their glass transition remained between $0-4{ }^{\circ} \mathrm{C}$ [56,57]. In the case of CNT-LCE composites, if the concentration of MWNTs, or SWNTs, is lower than $0.02 \mathrm{wt} \%$ then there is little change in the transition temperatures (even when there is no surfactant to help the dispersion) [52]. When the MWNT is well dispersed with the assistance of PyMC, the decreases in transition temperatures follow a systematic trend, with the nematic transition dropping from $74{ }^{\circ} \mathrm{C}$ for neat LCE to $72,69,68{ }^{\circ} \mathrm{C}$ respectively for $0.03,0.3$ and $1 \mathrm{wt} \% \mathrm{CNT}$ samples. However, when there is a higher amount of surfactant used to help the CNT dispersion, there is another mesophase transition at around $50{ }^{\circ} \mathrm{C}$ because of phase separation with the LCE matrix. Recently, it was reported that the addition of pristine SWNTs without any dispersant could induce a larger decrease in the transition temperature: from 82 to $63{ }^{\circ} \mathrm{C}$ for the sample with $1.8 \mathrm{wt} \%$ SWNT [63], probably due to phase separation between the CNT aggregates and the LCE matrix (nanotube demixing). In contrast, the SWNT-LCE composite prepared with conjugated polymers as a dispersant were rather inhomogeneous [69,70]. As seen from the optical image of a sample at $0.3 \mathrm{wt} \%$ concentration, it appears that the CNTs form large aggregates and phase separation is very clear. The authors concluded that the thermal stress of CNTs might account for the observed large decrease in the nematic transition point. These facts reinforce our view that re-aggregation of originally dispersed CNTs into less anisotropic clusters is very common, although not always paid attention to, and if this happens - the properties of the liquid crystalline phase are strongly disrupted.

Neat, mono-domain LCE can reversibly contract upon heating and extend with cooling. This thermal actuation effect is normally characterized by the extent to which the length changes between the isotropic phase (at high temperature) and the nematic phase (at ambient temperature). This is described as $\left(L-L_{0}\right) / L_{0}$, where $L$ is the length of a strip of the material at temperature $\mathrm{T}$, and $L_{0}$ is its length in the isotropic phase. No matter which particle processing methods, or particle concentrations were used, the resulting nanocomposites exhibited either unchanged or weakened thermal actuation. This was the case for nanoparticles forming a surface layer as well as nanoparticles homogeneously dispersed into the LCE matrix. There are two possible reasons for the weakening of the thermal actuation. Firstly, the nanoparticles may act as impurities, leading to a reduction in the LC nematic order (indicated 
by the decrease in $\mathrm{T}_{\mathrm{ni}}$, the isotropic-nematic transition temperature). Secondly, the addition of nanoparticles can increase the stiffness of the material, reducing the macroscopic elasticity (as has also been shown for other polymer systems). Li et al. [63] also verified that the tensile strength of the CNTLCE nanocomposites is significantly higher than those of neat LCEs. In addition, the rigidity of anisotropic nanoparticles that are aligned in LCEs may prevent the LCE matrix from undergoing full shape changes along the alignment axis during heating. For nanoparticles forming layers on the LCE surface, some groups report that there is no weakening of thermal actuation while some demonstrated an obvious weakening effect. Domenici et al. used centrifugation to form the conducting layers on the surface of the LCE [54,55]. $5 \mathrm{wt} \%$ of $\mathrm{PbTiO}_{3}$ nanoparticles in LCEs and $1 \mathrm{wt} \% \mathrm{MoO}_{3-x}$ nanowires reduced the elongation from $68 \%$ of the neat LCE to $55 \%$ and $58 \%$ respectively. This is different from the carbon reprocessed LCEs, which have almost the same thermal actuation as the neat LCE [75]. For the LCEs containing $\mathrm{Fe}_{3} \mathrm{O}_{4}$ nanoparticles, there is no systematic decrease with concentration, within the tested range from 0-1.4 vol\% [56]. All the samples show a similar contraction to that of the neat LCE, about $68.5 \%$ of the sample at room temperature and with good reproducibility. When a low concentration of MWNTs was mixed into LCE without any modification, "classical" thermal expansion experiments show that the thermal actuation of the nematic composite is qualitatively similar to the neat LCE, as shown in Figure 4. This composite has a constant sample length in the isotropic phase and a rapid elongation when the sample is cooled through the isotropic-nematic transition. However, the length change in this case is a little less than that observed in the neat LCE. Meanwhile, if the load applied to the material is held constant (in order to measure the strain at constant load), this quantity is lower in the composite than in the material without CNTs.

Figure 4. (a) The spontaneous length variation with temperature for LCE samples containing differing amounts of CNTs. At small concentrations CNTs do not significantly alter this thermal response (b) Azimuthal variation in X-ray intensity for samples containing increasing concentrations of MWCNTs: the addition of a small concentration of CNTs does not affect the internal orientational order of the LCE [47].
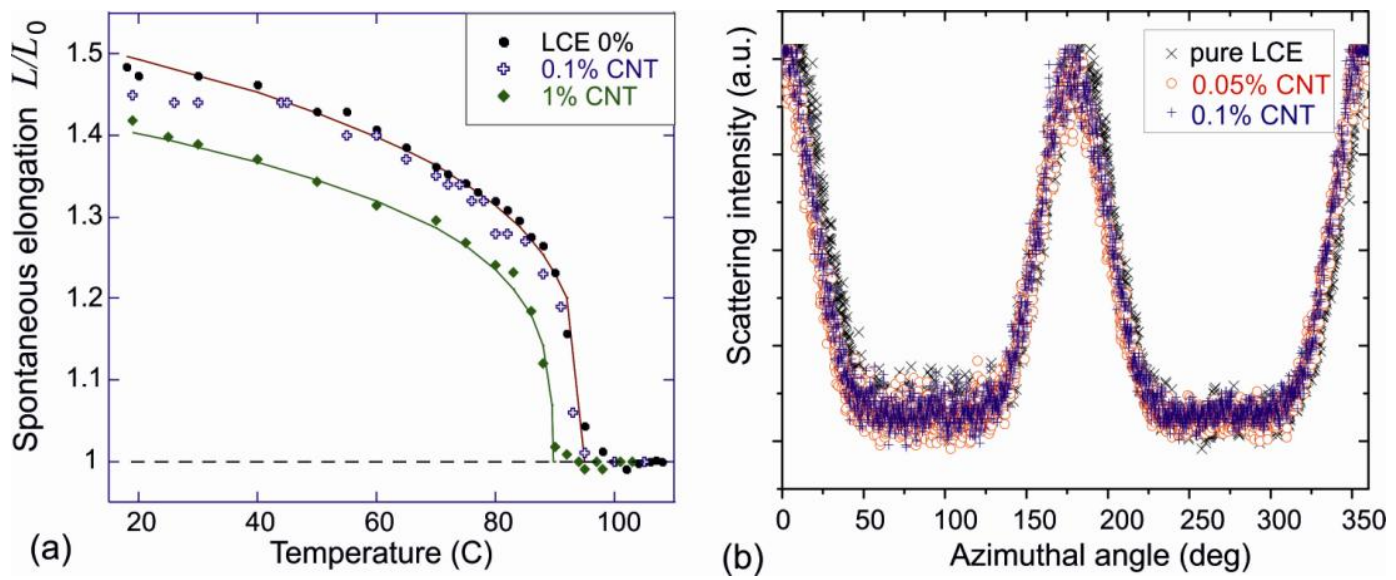

Good dispersion of CNTs may be helpful to counter this weakening in the thermal actuation effect. When MWNTs were dispersed in LCEs with surfactants, the thermal actuation remained almost unchanged until $1 \mathrm{wt} \%$ MWNTs were added [52]. The weakening of the thermal actuation becomes worse when a higher concentration of MWNTs is added (see the data for 1\% CNT in Figure 4(a)). 
A 3 wt $\%$ MWNT-LCE displayed a 14\% shape change compared with 50\% as was observed for the pure LCE. For SWNT-LCE composites, little information has been reported on the thermal actuation. In a recent case, the authors only reported the actuation of one sample with $0.3 \mathrm{wt} \%$ SWNTs. The length decreased from $4 \mathrm{~cm}$ to $2.9 \mathrm{~cm}$ [63]. However, no comparison was described between different CNT loadings and the neat LCE.

\subsection{Optical Properties and Photoactuation}

Both MWNTs and SWNTs absorb light very efficiently and convert the absorbed energy into heat [76]. In the visible/near-IR range, the absorption spectrum of MWNTs is featureless, whereas SWNTs show strong and specific absorptions [77]. Despite this difference, both could potentially act as nanoscale heating sources, changing photo-energy into heat. This fast and efficient energy transfer can trigger the LCE nematic-isotropic phase transition, leading to a shape change in the nanocomposite film. Yang et al. believe that in their SWNT-LCE system, the concentration of SWNTs $(0.01 \mathrm{wt} \%)$ is higher than the percolation limit [62]. Therefore, a SWNT percolation network exists in LCE. Such a network serves as a thermal conduction pathway due to the high thermal conductivity of the SWNTs. Heat was delivered through the LCE matrix uniformly and rapidly when an IR light source (intensity $45 \mathrm{~mW} / \mathrm{mm}^{2}$ at wavelength $808 \mathrm{~nm}$ ) was directed toward the material. Within $4 \mathrm{~s}$, the sample contracted to $80 \%$ of its maximum length. The temperature was well above the $T_{n i}$ immediately after high intensity IR irradiation. The full contraction was completed in $10 \mathrm{~s}$. When the light was switched off, the reverse process occurred, over a similar timescale. White light (intensity $230 \mathrm{~mW} / \mathrm{cm}^{2}$ ) was also applied in order to activate the SWNT-LCE nanocomposite [63]. The neat LCE shows little response to the light even when the light intensity reaches $10 \mathrm{~W} / \mathrm{cm}^{2}$. As in the case of the IR induced actuation reported by Yang et al., the SWCNT-LCE mono-domain film contracted immediately and reached maximum contraction after $10 \mathrm{~s}$ with a final temperature of $90{ }^{\circ} \mathrm{C}$ (which is above the nematic-isotropic transition). The reverse extension has a similar speed when the light was switched off. According to our experiments with light at an intensity of $1.5 \mathrm{~mW} / \mathrm{cm}^{2}$ at a wavelength of $675 \mathrm{~nm}$, the IR/visible actuation of aligned CNT composites is not confined to LCE systems; instead, it is a general phenomenon [65]. For "ordinary" (non-mesogenic) CNT-elastomer composites, non-aligned samples expand along their length while aligned samples exhibit a large uniaxial contraction upon IR irradiation. Such an opto-mechanical response was concluded to be mainly due to the contraction of CNTs after photon absorption rather than the pure heating effect induced by light [78]. The detailed mechanism has been discussed in $[65,72,78]$. A thermo-mechanical response induced by pure heating is present, but this was much slower and almost an order of magnitude smaller than the response to direct IR irradiation. In the mono-domain CNT-LCE nanocomposite, both CNT contraction and heating contribute to the observed optical actuation. After the subtraction of actuation induced by temperature increase, we could still observe significant contraction similar to the aligned CNT-PDMS samples with large pre-strains, which proved that CNT contraction after photo absorption is essential for the overall actuation of CNT-elastomer composites [65]. Due to this effect, the LCE-CNT samples could actuate well below the nematic-isotropic transition. This makes CNTs even more attractive than other nanoparticles for

applications where high temperature is undesirable. Alignment is of vital importance for the observed 
actuation. However, if there is no alignment of CNTs, the pure heating effect is the reason for the optoactuation as observed by Li et al. [63].

Parameters such as light density, preparation procedure, pre-strain, alignment quality, LCE chemical composition and so on, can affect the photoactuation achieved by CNT-LCE composites. Yang found that the actuation strain increased with the concentration of SWNTs [62]. When the IR light was switched on, the temperature of the SWNT-LCE films increased. The temperature increase was almost $15{ }^{\circ} \mathrm{C}$ higher for the $0.2 \mathrm{wt} \%$ sample than for that at $0.1 \mathrm{wt} \%$. More SWNTs in the matrix apparently produce more heat, resulting in faster actuation and larger axial strain. The sample with a concentration of $0.2 \mathrm{wt} \%$ SWNTs showed a faster response than that with a concentration of $0.1 \mathrm{wt} \%$, even when the CNTs were better aligned in the less concentrated sample. In the case of MWNTs, however, we unexpectedly observed that all samples with concentrations between $0.3-3 \mathrm{wt} \%$ showed about the same maximum actuation [52]. Actuation does not begin to decrease until the MWNT concentration is considerably lower than this.

It is hard to compare our work with that of other groups since so many parameters may be varied during the preparation of the nanocomposites. As expected, we found that the wavelength of light used has little effect on the photoactuation when MWNTs are embedded in the LCE matrix. For SWNTs, however, the wavelength may influence the actuation since it has been reported that a higher light intensity is required from an IR source than a white light source, in order to trigger this actuation. All experiments do show that light intensity plays an important role in the actuation, although the reported values of such intensity differ greatly. In the work of Yang et al. [62], when the intensity increases from $1.7 \mathrm{~W} / \mathrm{cm}^{2}$ to $3.4 \mathrm{~W} / \mathrm{cm}^{2}$, the contraction stress increased in the $0.3 \mathrm{wt} \%$ SNWT-LCE film from $55 \mathrm{kPa}$ to $80 \mathrm{kPa}$. In our work [52] a broad-peak light (675 nm peak) with intensity of $15 \mathrm{~mW} / \mathrm{cm}^{2}$ has produced the actuation stress of 6-8 $\mathrm{kPa}$. We believe these large discrepancies are due to the different levels of CNT dispersion in the elastomer matrix.

As shown in Figure 5(b), the higher the light intensity, the stronger the actuation stress that is achieved by the material. Other factors also affect this actuation. For example, higher crosslinking density will impede the actuation process by increasing the elastic modulus of the material and preventing the relative motion of filler particles.

Besides absorbing visible and near-IR light, CNTs also show absorption within the microwave, terahertz and megahertz frequency ranges [79]. The high loading and alignment of CNTs also makes LCE composites potentially anisotropic at these frequencies. Using Terahertz spectroscopy as an example, we measured the $\mathrm{THz}$ transmission time-domain spectra of CNT-LCE composites in a frequency range of 0.2-3.5 THz. As shown in Figure 5(c), the addition of CNTs to LCEs greatly enhanced the $\mathrm{THz}$ absorption, especially in the higher-frequency region. When the concentration of CNTs is high enough (>1 wt \%), the absorption coefficient parallel to the CNT alignment axis becomes much larger than that in the perpendicular direction; $3 \mathrm{wt} \%$ data is shown in Figure 5(c). The corresponding refractive index data show a similar anisotropic tendency (Figure 5(d)). Such anisotropic optical properties, in addition to the LCEs' actuating behavior, make these materials attractive candidates for the preparation of tunable polarizers, wave guides, or other optoelectronic devices. 
Figure 5. (a) Infrared photo-induced actuation in constant-strain geometry, for the samples labelled on the plot; (b) The influence of light intensity from a monochromatic light source, showing that the intensity has a considerable effect on the maximum actuation achieved; (c) THz time-domain spectroscopy: absorption spectra of a blank, $0.3 \%$ and $3 \mathrm{wt} \%$ CNT composites when the $\mathrm{THz}$ polarization is parallel, //, and perpendicular, + , to the direction of LCEs; (d) The absorption coefficient and the refractive index of samples with different CNT concentrations at $2 \mathrm{THz}$. Uniaxial thermal expansion of nematic elastomers: the comparison between the neat network $(\bullet)$ and the $0.02 \%$ CNT composite $\left({ }^{\circ}\right)$.
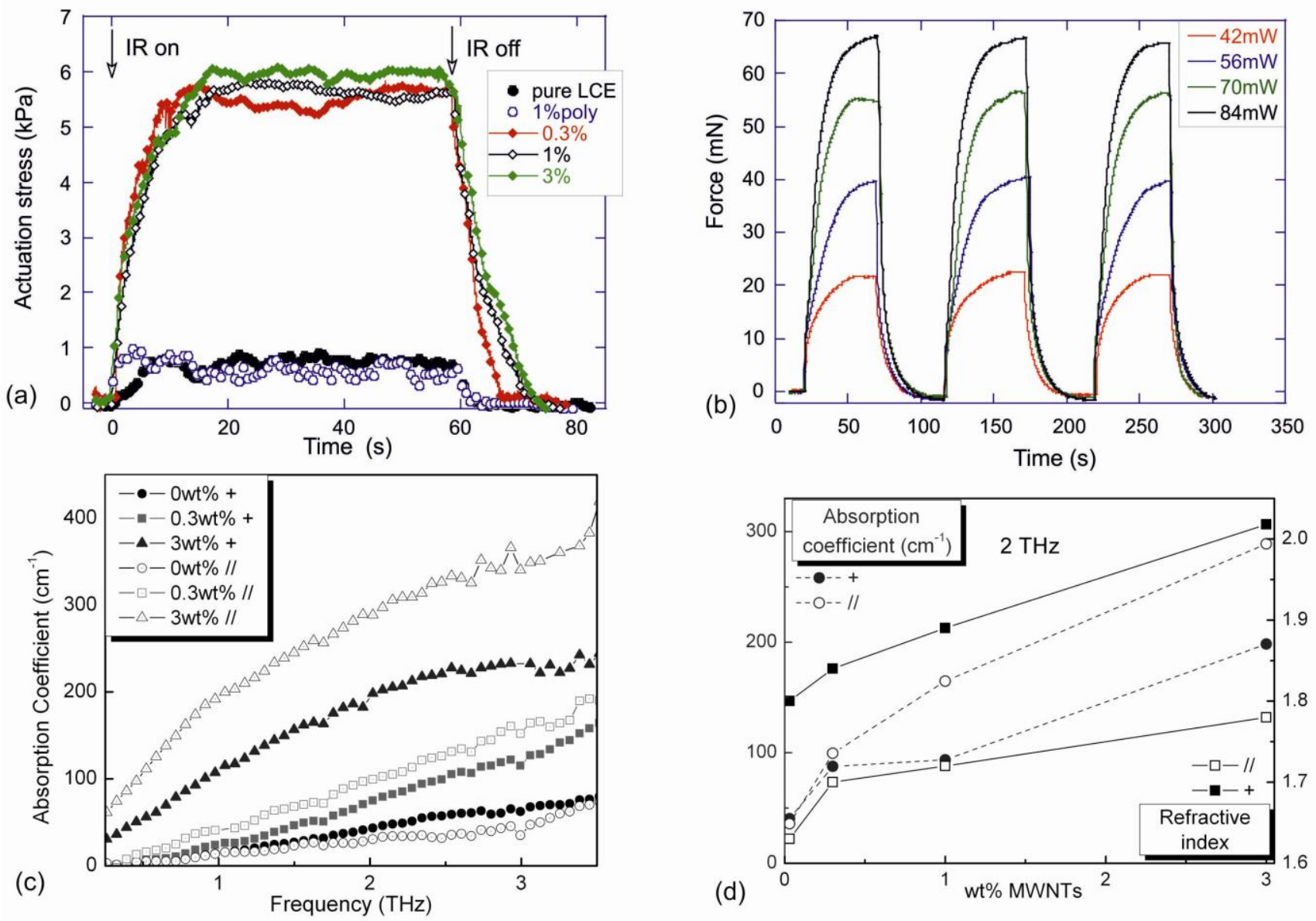

\subsection{Electrical Actuation}

The incorporation of nanoparticles enables the LCEs to usefully respond to electric fields, while the ordinary (neat) LCE can only do so in the "soft-elasticity" regime when the sample is completely mechanically unconstrained [80,81]. When a conducting layer is formed by nanoparticles in LCEs, the LCEs exhibit electromechanical actuation because of Joule heating when an electric current passes through the material. Chambers et al. have made a thorough investigation of the electromechanical actuation of carbon processed LCEs. Besides the conducting layer formed in an LCE by carbon nanohorns and carbon nanoparticles, or sprayed carbon coatings, other conducting layers in LCEs were also prepared by $\mathrm{PbTiO}_{3}$ and $\mathrm{MoO}_{3-x}$ nanowires. Studies of the electromechanical actuation of these materials have not yet been attempted. Since electromechanical actuation induced by a conducting layer due to Joule heating has been reviewed in [43], we here focus our attention on another mechanism, that is, the actuation due to electrostatic fields. Such actuation was demonstrated using 
mono-domain LCEs with CNTs [53]. CNTs are conducting materials, but their electromechanical response could have a very different mechanism from resistive heating, as has been demonstrated in carbon coated or reprocessed LCEs [44-46]. We used MWNTs at very low concentrations (far below the percolation limit). The LCE-CNT composites were in fact electrically insulating. We assume that the mechanism of the response originates from the CNTs' large anisotropy in terms of their polarizability and dielectric constant. When CNTs are exposed to an electric field whose direction is perpendicular to the CNT length axis, they are immediately polarized, resulting in a high local torque which drives CNTs to rotate in order to be aligned with the direction of the electric field. The rotational force experienced by the CNTs is transferred to the surrounding elastic network, producing a measurable stress within the LCE and a resulting mechanical contraction. The electric field used to trigger the actuation was very high $\mathrm{E} \approx 1.9 \times 10^{6} \mathrm{~V} / \mathrm{m}$ because there was less than $0.02 \mathrm{wt} \%$ of carbon nanotubes in the LCEs.

Figure 6. (a) Dependence of the maximum actuation stress $\sigma_{\max }$ on the applied field strength, for the $0.02 \mathrm{wt} \%$ CNT sample. The inset shows the elapsed time dependence, with each cycle at increasing constant field E. (b) The response to an alternating field, switching on and off every $30 \mathrm{~s}$, demonstrating the overall stability of the response. The inset shows the short initial period of this test, showing the high speed of the response.
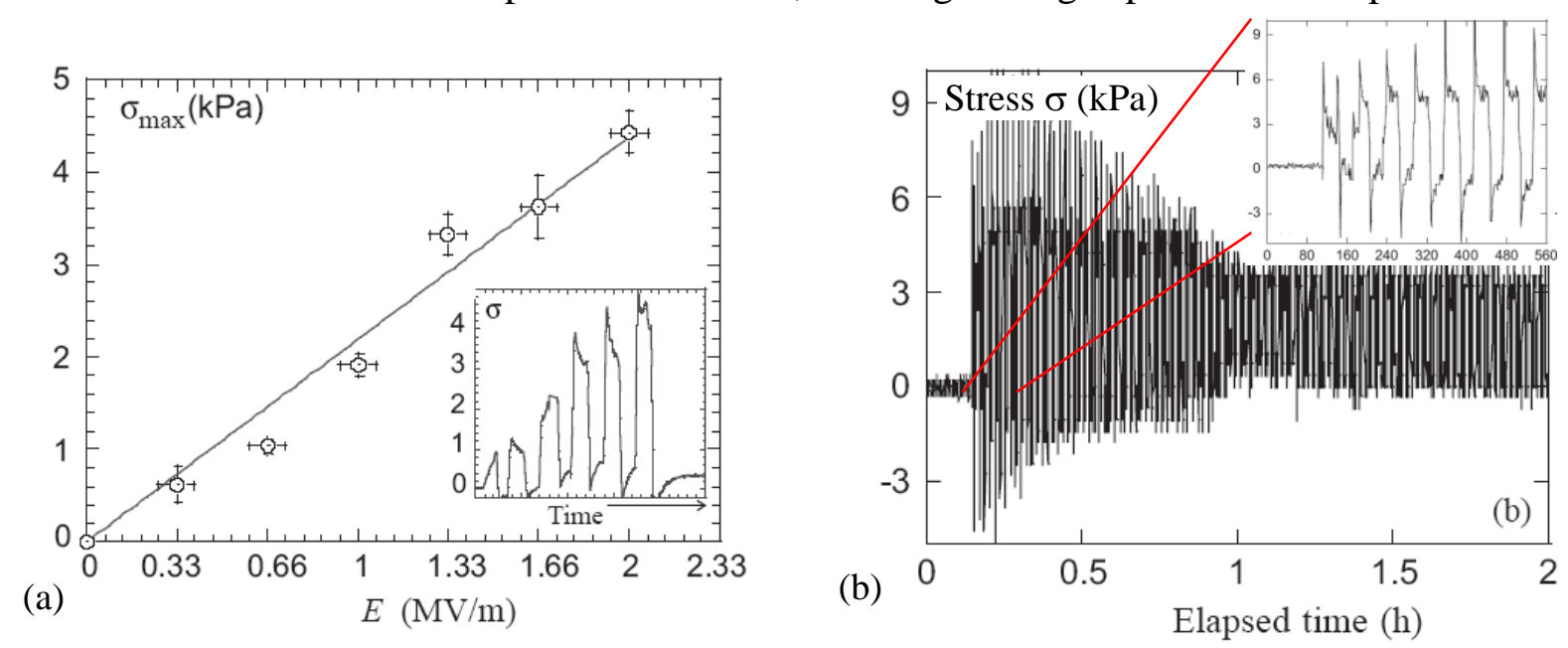

As shown in Figure 6(a), once the electric field is switched on, the stress exerted by the film increases rapidly and reaches a plateau. The maximum stress reached $\left(\sigma_{\max }\right)$ depends on CNT concentration, and is of the order of $1 \mathrm{kPa}$ (with a corresponding strain of $0.1 \%$ ). This plateau level also follows a linear dependence on the applied field, Figure 6(b). This electromechanical response was not found in the neat LCEs without nanotubes. The MWNT-LCE also allows good reproducibility and fast response time. As shown in Figure 6(b), the 0.02\% CNT-LCE material responds very fast to both the "on" and "off" cycles when the electric field is switched on and off every $30 \mathrm{~s}$. Meanwhile, both the response speed and the plateau stress amplitude have very good reproducibility. All of the above is quite encouraging in the context of practical applications for this material. However, this system is far from well understood. For example, the stress in the off-cycle did not go back to the initial zero level within the measured (long) time span under the applied field; while in the fastalternating field experiment there is no residual stress in the field-off state; the exerted stress $\sigma_{\max }$ is 
linearly dependent on the electric field while it should be expected to follow $E^{4}$. Although further study would be required in order to fully understand the system, there is no doubt that the CNT-LCE material provides an opportunity to access electrically driven actuation. As we have shown, higher concentrations of CNTs are expected to offer higher electric energy densities. This is also indicated by our later work with high loading of CNTs in the composite materials.

Figure 7. Complex resistivity against frequency, for CNT-LCE composites of different CNT loading. The insert compares the scaling behavior on a log-log scale with that of neat PDMS.

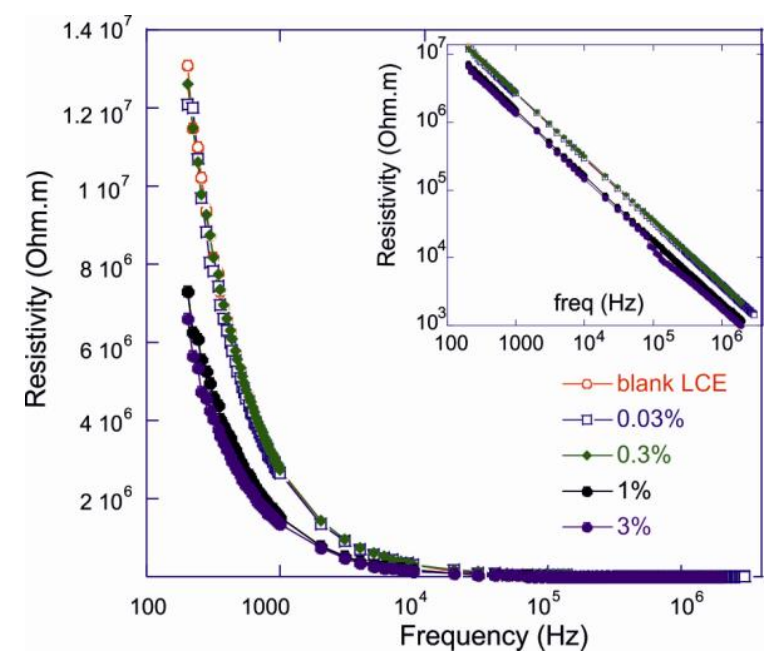

\subsection{Magnetic Actuation}

It is well-known that magnetic nanoparticles can be manipulated by an alternating (high frequency) magnetic field. If the suspended nanoparticles have the freedom to move, the movement gives rise to viscous heating of the surrounding matrix. Meanwhile, the Neel relaxation of the internal magnetic moment acts as another way of heat generation. Therefore, magnetic nanoparticles absorb electromagnetic energy and transfer it into heat. For the LCE system, Levine and others have proposed that Neel relaxation is the major reason for the heating effect since the nanoparticles have lost their mobility due to the confinement of the matrix $[82,83]$. According to a multi-scale model, upon the application of the external magnetic field the Neel relaxation firstly heats up the nanoparticles, leading to temperature gradients within them. Then heat is released into the surrounding LCE matrix, accompanied by the appearance of a large temperature gradient in the LCE matrix and a decrease in the temperature gradient within the nanoparticles. Such nanoscale heat transfer happens very quickly, in the order of few microseconds. Later, the temperature gradients fade away and the entire matrix may be considered as reaching a uniform temperature. Longer exposure to the magnetic field leads to temperature increases which induce thermal phase transitions of the LCE. As a result, actuation of the mono-domain LCEs could be triggered by magnetic fields.

Experimental results fit well with this theory. The incorporation of magnetic nanoparticles as nano-scale heat sources leads to a faster response and remote control of the actuation. Without the magnetic nanoparticles, the neat LCE shows little contraction in a magnetic field. To obtain effective actuation, the concentration of incorporated magnetic nanoparticles must be sufficiently high to generate 
enough heat to trigger the phase transition. In Kaiser's experiment, the concentration of $\mathrm{Fe}_{3} \mathrm{O}_{4}$ must be above 1.4 vol\% [56]. Due to the hysteresis and relaxation processes that occur during electromagnetic irradiation, the heat transferred into the LCE increases the temperature while neat LCE undergoes little temperature change under the same conditions. It takes a couple of minutes for the material to reach the saturation length, which decreases as the nanoparticle concentration increases. In the best case, for the high concentration 1.64 vol\% of $\mathrm{Fe}_{3} \mathrm{O}_{4}$, the reversible shape change is about 27\%, which is almost the same as the thermal actuation of neat LCEs without any nanoparticles. This also shows that actuation depends on the strength of the magnetic field. This is easy to understand since a magnetic field with higher strength provides more energy and therefore a larger temperature increase. Therefore, it is possible to control the final length of the LCE by changing the amplitude of the magnetic field used. The temperature increase follows a logarithmic function with time. Not all of the energy accumulated by the nanoparticles has been used to heat up the samples. Instead some is lost because of heat exchange between the sample and the environment. Meanwhile, this magnetic field induced actuation still suffers from low efficiency, and the response time needs further improvement in order for this material to be a practical actuator. A possible improvement to this system may rely on anisotropic magnetic nanoparticles which have stronger affinity with the LCE matrix [84].

\section{Concluding Remarks}

Benefiting from the "boom" in nanoscience and nanotechnology research, the incorporation of nanoparticles into LCEs has been identified as an effective strategy for the fabrication of novel functional composites and smart devices with advanced performance. The electronic, magnetic and optical properties of the nanoparticles have paved the way to new and improved properties of LCE composites. For practical applications of LCE materials, it would be highly beneficial to have flexible control over the actuation, by external stimuli rather than direct heating. This requirement could be satisfied by incorporating nanoparticles (acting as local heat generators) into an LCE matrix in order to produce LCE composites that can be controlled remotely with fast actuation. Although the processing of these materials remains difficult, it has been demonstrated that contactless actuation is achievable using electricity, magnetism and light. This actuation is fully reversible and relatively fast; "neat" LCEs (without added nanoparticles) show actuation in response to heat only.

One critical challenge in the fabrication of LCE nanocomposites is to make sure that the addition of nanoparticles preserves the LC properties and thermomechanical capability of the polymer, while introducing new modes of actuation and quick response times. Efficient processing of these materials is the key to achieving useful mono-domain LCE nanocomposites. Firstly, the concentration of nanoparticles must be optimized. Too few nanoparticles in the material may fail to trigger actuation, while too many may destroy the liquid crystalline properties of the LCE. Secondly, modification of the nanoparticles is usually necessary to ensure the homogeneous dispersion of nanoparticles in LCEs. The standard two-step crosslinking method is a widely used procedure to prepare mono-domain LCEs. Using this method, nanoparticles can be introduced as one component of the reaction mixture, and must therefore initially be dispersed in solution. While mixing techniques such as mechanical shearing and sonication can improve the dispersion, it is best to modify the surface of the nanoparticles in order to obtain well-separated nanoparticles. This modification can also promote good interfacial adhesion 
between nanoparticles and the LCE matrix; this leads to efficient stress transfer, makes the processing of the materials more straightforward, and greatly improves the properties of the final composites. In situ nanoparticle generation may be an alternative for the formation of aggregation-free LCE nanocomposites, because many nanoparticle precursors are readily soluble in organic solvents. If the precursor (normally metal ions) could be reduced during or after the LCE reaction, the nanoparticles could be trapped within the LCE matrix (thus avoiding the possibility of aggregate formation).

Attaching nanoparticles to the LCE could possibly be another way to obtain homogenous LCE nanocomposites. Magnetic nanoparticles have been introduced into LC networks to act as both fillers and crosslinkers. Dopamine-functionalized ferrite nanoparticles were covalently incorporated into the LCE as cross-linkers by Song et al. [85]. The oleate-coated iron oxide nanoparticles were synthesized first, then the oleate groups were partially replaced with reactive vinyl groups that could react with the polysiloxane polymer backbone. The inclusion of these ferrite nanoparticles $(6 \mathrm{~nm})$ showed super paramagnetic behavior at room temperature. However, mono-domain elastomeric polymers have not yet been fabricated using this method. Similarly, Garcia-Marquez introduced iron oxide nanoparticles into a smectic main-chain LC polymer; this also shows low-temperature ferrimagnetism [86]. Again, this method has yet to be used to generate mono-domain LCEs.

The alignment of anisotropic nanoparticles in LCEs is worthy of further attention. On the one hand, it is anticipated that better alignment of anisotropic filler particles will considerably improve the performance of LCEs. However, the exploration of anisotropic nanoparticles is often more focused on control of their spatial ordering, which is essential but extremely challenging for many applications. Compared to un-oriented materials, the aligned samples could offer inviting anisotropic electrical, optical and magnetic properties, as is illustrated by the anisotropic THz response of a CNT-LCE composite material. Importantly, anisotropic nanoparticles may also offer an opportunity to prepare mono-domain LCEs using relatively low electric and magnetic fields. It has been well established that stretching in the two-step cross-linking method encourages anisotropic nanoparticles to align along the stretching direction. Besides stretching, alignment of mesogens by electric/magnetic fields also works very well, but powerful field strengths are necessary. As has been proved by both experimental and theoretical work [87] with low molecular mass liquid crystals, the coupling effect between nanoparticles and LCs could be substantially enhanced by using rod shaped nanoparticles; these provide strong anchoring to the LCs and guarantee a large torque. Recently, it was shown that during the in situ generation of Co-nanorods in a liquid crystalline polymer, a magnetic field with an exceptionally low strength is sufficient to align the nanorods [88]. The alignment of the LC mesogens also improves because of the co-operative effects of LCP and nanorod alignment. Therefore, it is expected that the alignment of mesogens, assisted by nanoparticle orientation controlled by a magnetic field, could reduce the necessary strength of the external field by exploiting the coupling between the LCE ordered matrix and the nanoparticles.

Despite stimulating progress in LCE nanocomposites, this field is still young and much work must still be done in order to fabricate ideal stimuli-responsive materials for practical applications. Considerable challenges await researchers in this research topic. For example, the processing is difficult; it is hard to optimize various factors at the nano-scale in order to balance the overall macroscopic performance; few studies have addressed the effects of nanoparticle dimension, purity and polydispersity; the ability to orientate nanoparticles has not yet been fully explored; and the mechanism 
of actuation by remote stimuli needs to be investigated further both theoretically and experimentally, in order to translate specific guidelines for rational design and optimization of the LCE nanocomposite into outstanding performance. Progress in this area is dependent on collaborative, multidisciplinary research at the boundaries between chemistry, physics and engineering. However, there is no doubt that the consolidation of nanoparticles and LCEs has emerged as another promising tool for the application of LCEs in practical functional devices as multi-stimuli responsive soft composites with excellent mechanical flexibility and durability.

\section{Acknowledgments}

This work has been supported by EU FP7 funding program under contract NMP 228916 "Nano-Optical Mechanical Systems (NOMS)". We thank Y.Y. Huang for help and advice on many aspects of this work, and this review.

\section{References}

1. Ionov, L. Actively-moving materials based on stimuli-responsive polymers. J. Mater. Chem. 2010, 20, 3382-3390.

2. Lendlein, A. Progress in actively moving polymers. J. Mater. Chem. 2010, 20, 3332-3334.

3. Warner, M.; Terentjev, E.M. Liquid Crystal Elastomers; Oxford University Press: Oxford, UK, 2007.

4. Yamada, M.; Kondo, M.; Mamiya, J.I.; Yu, Y.L.; Kinoshita, M.; Barrett, C.J.; Ikeda, T. Photomobile polymer materials: Towards light-driven plastic motors. Angew. Chem. Int. Ed. Engl. 2008, 47, 4986-4988.

5. Ohm, C.; Brehmer, M.; Zentel, R. Liquid crystalline elastomers as actuators and sensors. Adv. Mater. 2010, 22, 3366-3387.

6. Amigó-Melchior, A.; Finkelmann, H. A concept for bifocal contact- or intraocular lenses: Liquid single crystal hydrogels (“LSCH”). Polym. Adv. Technol. 2002, 13, 363-369.

7. Sánchez-Ferrer, A.; Fischl, T.; Stubenrauch, M.; Wurmus, H.; Hoffmann, M.; Finkelmann, H. Photo-crosslinked side-chain liquid-crystalline elastomers for microsystems. Macromol. Chem. Phys. 2009, 210, 1671-1677.

8. Sánchez-Ferrer, A.; Fischl, T.; Stubenrauch, M.; Albrecht, A.; Wurmus, H.; Hoffmann, M.; Finkelmann, H. Liquid-crystalline elastomer microvalve for microfluidics. Adv. Mater. 2011, 23, 4526-4530.

9. Finkelmann, H.; Kim, S.T.; Muæoz, A.; Palffy-Muhoray, P.; Taheri, B. Tunable mirrorless lasing in cholesteric liquid crystalline elastomers. Adv. Mater. 2001, 13, 1069-1072.

10. Hirota, Y.; Ji, Y.; Serra, F.; Tajbakhsh, A.R.; Terentjev, E.M. Effect of crosslinking on the photonic bandgap in deformable cholesteric elastomers. Opt. Express 2008, 16, 5320-5331.

11. Schmidtke, J.; Stille, W.; Finkelmann, H.; Kim, S.T. Laser emission in a dye doped cholesteric polymer network. Adv. Mater. 2002, 14, 746-749.

12. Schmidtke, J.; Stille, W.; Finkelmann, H. Defect mode emission of a dye doped cholesteric polymer network. Phys. Rev. Lett. 2003, doi: 10.1103/PhysRevLett.90.083902. 
13. Schmidtke, J.; Kniesel, S.; Finkelmann, H. Probing the photonic properties of a cholesteric elastomer under biaxial stress. Macromolecules 2005, 38, 1357-1363.

14. Fridrikh, S.V.; Terentjev, E.M. Polydomain-monodomain transition in nematic elastomers. Phys. Rev. E 1999, 60, 1847-1857.

15. Kremer, F.; Lehmann, W.; Skupin, H.; Hartmann, L.; Stein, P.; Finkelmann, H. Piezoelectricity in ferroelectric liquid crystalline elastomers. Polym. Adv. Technol. 1998, 9, 672-677.

16. Nishikawa, N.; Finkelmann, H. Smectic-A-liquid single crystal elastomers-Strain induced break-down of smectic layers. Macromol. Chem. Phys. 1999, 200, 312-322.

17. Sánchez-Ferrer, A.; Finkelmann, H. Polydomain-monodomain orientational process in smectic-C main-chain liquid-crystalline elastomers. Macromol. Rapid Commun. 2011, 32, 309-315.

18. Kramer, D.; Finkelmann, D. Mechanically induced tilt in smectic-A elastomers. Soft Matter 2011, 7, 1861-1867.

19. de Gennes, P.-G.; Hébert, M.; Kant, R. Artificial muscles based on nematic gels. Macromol. Symp. 1997, 113, 39-49.

20. de Gennes, P.-G. A semi-fast artificial muscle. C.R. Acad. Sci. Ser. IIB Mech. Phys. Chem. Astron. 1997, 324, 343-348.

21. Küpfer, J.; Finkelmann, H. Liquid crystal elastomers: Influence of the orientational distribution of the crosslinks on the phase behaviour and reorientation processes. Macromol. Chem. Phys. 1994, 159, 1353-1367.

22. Tajbakhsh, A.R.; Terentjev, E.M. Spontaneous thermal expansion of nematic elastomers. Eur. Phys. J. E. 2001, 6, 181-188.

23. Finkelmann, H.; Nishikawa, E.; Pereira, G.G.; Warner, M. A new opto-mechanical effect in solids. Phys. Rev. Lett. 2001, 87, 015501:1-015501:4.

24. Hogan, P.M.; Tajbakhsh, A.R.; Terentjev, E.M. UV manipulation of order and macroscopic shape in nematic elastomers. Phys. Rev. E 2002, 65, 041720:1-041720:10.

25. Camacho-Lopez, M.; Finkelmann, H.; Palffy-Muhoray, P.; Shelley, M.; Fast liquid-crystal elastomer swims into the dark. Nat. Mater. 2004, 3, 307-310.

26. Ikeda, T.; Mamiya, J.; Yu, Y. Photomechanics of liquid-crystalline elastomers and other polymers. Angew. Chem. Int. Ed. 2007, 46, 506-528.

27. Li, C.S.; Lo, C.W.; Zhu, D.F.; Li, C.H.; Liu, Y.; Jiang, H.R. Synthesis of a photoresponsive liquid-crystalline polymer containing azobenzene. Macromol. Rapid Commun. 2009, 30, 1928-1935.

28. Yu, Y.; Nakano, M.; Ikeda, T. Photomechanics: Directed bending of a polymer film by light. Nature 2003, doi: 10.1038/425145a.

29. Harvey, C.L.; Terentjev, E.M. Role of polarization and alignment in photoactuation of nematic elastomers. Eur. Phys. J. E 2007, 23,185-189.

30. Sánchez-Ferrer, A.; Merekalov, A.; Finkelmann, H. Opto-mechanical effect in potoactive nematic side-chain liquid-crystalline elastomers. Macromol. Rapid Commun. 2011, 32, 672-678.

31. Sánchez-Ferrer, A. Light-induced disorder in liquid-crystalline elastomers for actuation. Proc. SPIE 2011, 8107, 810702:1-810702:8.

32. Garcia-Amorós, J.; Piñol, A.; Finkelmann, H.; Velasco, D. Azophenol-based liquid-crystalline elastomers for light-driven actuators. Org. Lett. 2011, 13, 2282-2285. 
33. Garcia-Amorós, J.; Finkelmann, H.; Velasco, D. Influence of the photo-active azo cross-linker spacer on the opto-mechanics of polysiloxane elastomer actuators. J. Mater. Chem. 2011, 21, 1094-1101.

34. Cviklinski, J.; Tajbakhsh, A.R.; Terentjev, E.M. UV isomerisation in nematic elastomers as a route to photo-mechanical transducer. Eur. Phys. J. E 2002, 9, 427-434.

35. Lehmann, W.; Skupin, H.; Tolksdorf, C.; Gebhard, E.; Zentel, R.; Krüger, P.; Lösche1, M.; Kremer, F. Giant lateral electrostriction in ferroelectric liquid-crystalline elastomers. Nature 2001, 410, 447-450.

36. Na, Y.H.; Aburaya, Y.; Orihara, H.; Hiraoka, K. Measurement of electrically induced shear strain in a chiral smectic liquid-crystal elastomer. Phys. Rev. E 2011, 83, 061709:1-061709:5.

37. Papadopoulos, P.; Heinze, P.; Finkelmann, H.; Kremer, F. Electromechanical properties of smectic C* liquid crystal elastomers under shear. Macromolecules 2010, 43, 6666-6670.

38. Verduzco, R.; Luchette, P.; Hong, S.H.; Harden, J.; DiMasi, E.; Palffy-Muhoray, P.; Kilbey, S.M., II; Sprunt, S.; Gleeson, J.T.; Jákli, A. Bent-core liquid crystal elastomers. J. Mater. Chem. 2010, 20, 8488-8495.

39. Harden, J.; Chambers, M.; Verduzco, R.; Luchette, P.; Gleeson, J.T.; Sprunt, S.; Jákli, A. Giant flexoelectricity in bent-core nematic liquid crystal elastomers. Appl. Phys. Lett. 2010, 96, 102907:1-102907:3.

40. Heinze, P.; Finkelmann, H. Shear deformation and ferroelectricity in chiral SmC* main-chain elastomers. Macromolecules 2010, 43, 6655-6665.

41. Huang, Y.Y.; Biggins, J.; Ji, Y.; Terentjev, E.M. Mechanical bistability in liquid crystal elastomer-wire composite actuators. J. Appl. Phys. 2010, 107, 083515:1-083515:8.

42. Shenoy, D.K.; Laurence Thomsen, D., III.; Srinivasan, A.; Keller, P.; Ratna, B.R. Carbon coated liquid crystal elastomer film for artificial muscle applications. Sens. Actuat. A Phys. 2002, 96, 184-188.

43. Chambers, M.; Finkelmann, H.; Remškar, M.; Sánchez-Ferrer,A.; Zalar, B.; Žumer, S. Liquid crystal elastomer-nanoparticle systems for actuation. J. Mater. Chem. 2009, 19, 1524-1531.

44. Chambers, M.; Zalar, B.; Remskar, M.; Zumer, S.; Finkelmann, H. Actuation of liquid crystal elastomers reprocessed with carbon nanoparticles. Appl. Phys. Lett. 2006, 89, 243116:1-243116:3.

45. Chambers, M. Zalar, B. Remskar, M. Kovac, J. Finkelmann, H. Zumer, S. Investigations on an integrated conducting nanoparticle-liquid crystal elastomer layer. Nanotechnology 2007, doi: 10.1088/0957-4484/18/41/415706.

46. Chambers M, Zalar B, Remškar M, Finkelmann H, Zumer, S. Piezoresistivity and electro-thermomechanical degradation of a conducting layer of nanoparticles integrated at the liquid crystal elastomer surface. Nanotechnology 2008, doi: 10.1088/0957-4484/19/15/155501.

47. Marshall, J.E.; Ji, Y.; Torras, N.; Zinoviev, K.; Terentjev, E.M. Carbon-nanotube sensitized nematic elastomer composites for IR-visible photo-actuation. Soft Matter 2012, 8, 1570-1574

48. Huang, Y.Y.; Terentjev, E.M. Tailoring the electrical properties of carbon nanotube-polymer composites. Adv. Funct. Mater. 2010, 20, 4062-4068.

49. Ajayan, P.M.; Tour, J. Nanotube composites, Nature 2007, 447, 1066-1068.

50. Dierking, I.; Scalia, G.; Morales, P.; LeClere, D. Aligning and reorienting carbon nanotubes with nematic liquid crystals. Adv. Mater. 2004, 16, 865-869. 
51. Ji, Y.; Huang, Y.Y.; Terentjev, E.M. Dissolving and aligning carbon nanotubes in thermotropic liquid crystals. Langmuir 2011, 27, 13254-13260.

52. Ji, Y.; Huang, Y.Y.; Rungsawang, R.; Terentjev, E.M. Dispersion and alignment of carbon nanotubes in liquid crystalline polymers and elastomers. Adv. Mater. 2010, 22, 3436-3440.

53. Courty, S.; Mine, J.; Tajbakhsh, A.R.; Terentjev. Nematic elastomers with aligned carbon nanotubes: New electromechanical actuators. Europhys. Lett. 2003, 64, 654-660.

54. Domenici, V.; Conradi, M.; Remškar, M.; Viršek, M.; Zupančič, B.; Mrzel, A.; Chambers, M.; Zalar, B. New composite films based on $\mathrm{MoO}_{3}-\mathrm{X}$ nanowires aligned in a liquid single crystal elastomer matrix. J. Mater. Sci. 2011, 46, 3639-3645.

55. Domenici, V.; Zupančič, B.; Laguta, V.V.; Belous, A.G.; V’yunov, O.I.; Remškar, M.; Zalar, B. $\mathrm{PbTiO}_{3}$ nanoparticles embedded in a liquid crystalline elastomer Matrix: Structural and ordering properties. J. Phys. Chem. C 2010, 114, 10782-1078.

56. Kaiser, A.; Winkler, M.; Krause, S.; Finkelmann, H.; Schmidt, A.M. Magnetoactive liquid crystal elastomer nanocomposites. J. Mater. Chem. 2009, 19, 538-543.

57. Winkler, M.A.; Krause, S.; Finkelmann, H.; Schmidt, A.M. Liquid crystal eastomers with magnetic actuation. Macromol. Symp. 2010, 291-292, 186-192.

58. Zentel, R.; Reckert, G. Liquid crystalline elastomers based on liquid crystalline side group, main chain and combined polymers. Macromol. Chem. 1986, 187, 1915-1920.

59. Legge, C.H.; Davis, F.J.; Mitchell, G.R. Memory effects in liquid crystal elastomers. J. Phys. II 1991, $1,1253-1261$.

60. Urayama, K. Selected issues in liquid crystal elastomers and gels. Macromolecules 2007, 40, 2277-2288.

61. Thomsen III, D.L.; Keller, P.; Naciri, J.; Pink, R.; Jeon, H.; Shenoy, D.; Ratna, B.R.; Liquid crystal elastomers with mechanical properties of a muscle. Macromolecules 2001, 34, 5868-5875.

62. Yang, L.; Setyowati, K.; Li, A.; Gong, S.; Chen, J. Reversible infrared actuation of carbon nanotube-liquid crystalline elastomer nanocomposites. Adv. Mater. 2008, 20, 2271-2275.

63. Li, C.; Liu, Y.; Lob, C.; Jiang, H. Reversible white-light actuation of carbon nanotube incorporated liquid crystalline elastomer nanocomposites. Soft Matter 2011, 7, 7511-7516.

64. Velasco-Santos, C.; Martinez-Hernandez, A.L.; Castano, V.M. Carbon nanotube-polymer nanocomposites: The role of interfaces. Compos. Interfaces 2005, 11, 567-586.

65. Ahir, S.V.; Squires, A.M.; Tajbakhsh, A.R.; Terentjev, E.M. Infrared actuation in aligned polymer-nanotube composites. Phys. Rev. B 2006, 73, 085420:1-085420:12.

66. Zou, J.; Liu, L.; Chen, H.; Khondaker, S.I.; McCullough, R.D.; Huo, Q.; Zhai, L. Dispersion of pristine carbon nanotubes using conjugated block copolymers. Adv. Mater. 2008, 20, 2055-2060.

67. Manivannan, S.; Jeong, I.O.; Ryu, J.H.; Lee, C.S.; Kim, K.S.; Jang, J.; Park, K.C. Dispersion of single-walled carbon nanotubes in aqueous and organic solvents through a polymer wrapping functionalization. J. Mater. Sci. Mater. Electron. 2009, 20, 223-229.

68. Datsyuk, V.; Landois, P.; Fitremann, J.; Peigney, A.; Galibert, A.M.; Soula, B.; Flahaut, E. Double-walled carbon nanotube dispersion via surfactant substitution. J. Mater. Chem. 2009, 19, 2729-2736. 
69. Kang, Y.K.; Lee, O.; Deria, P.; Kim, S.H.; Park, T.; Bonnell, D.A.; Saven, J.G.; Therien, M.J. Helical wrapping of single-walled carbon nanotubes by water soluble poly(p-phenyleneethynylene). Nano Lett. 2009, 9, 1414-1418.

70. Chen, J.; Liu, H.; Weimer, W.A.; Halls, M.D.; Waldeck, D.H.; Walker, G.C. Noncovalent engineering of carbon nanotube surfaces by rigid, functional conjugated polymers. J. Am. Chem. Soc. 2002, 124, 9034-9035.

71. Sánchez-Ferrer, A.; Mezzenga, R.; Dietsch, H. Orientational behavior of ellipsoidal silica-coated hematite nanoparticles integrated within an elastomeric matrix and its mechanical reinforcement. Macromol. Chem. Phys. 2011, 212, 627-634.

72. Ahir, S.V.; Terentjev, E.M. Photomechanical actuation in polymer nanotube composites. Nat. Mater. 2005, 4, 491-495.

73. Scalia, G. Alignment of carbon nanotubes in thermotropic and lyotropic liquid crystals. Chem. Phys. Chem. 2010, 11, 333-340.

74. Yu, G.; Cao, A.; Lieber, C.M. Large-area blown bubble films of aligned nanowires and carbon nanotubes. Nat. Nanotechnol. 2007, 2, 372-377.

75. Zumer, S.; Chambers, M.; Cordoyiannis, G.; Finkelmann, H.; Kutnjak, Z.; Lebar, A.; Remskar, M.; Zalar, B. Some advances in liquid crystal elastomers: From crosslinks affected ordering to carbon nanoparticles enabled actuation. MRS Proc. 2007, 1005, 1005-Q04-06:1-1005-Q04-06:11.

76. Ajayan, P.M.; Terrones, M.; de la Guardia, A.; Huc, V.; Grobert, N.; Wei, B.Q.; Lezec, H.; Ramanath, G.; Ebbesen, T.W. Nanotubes in a flash--ignition and reconstruction. Science 2002, 296, 705.

77. Hamon, M.A.; Itkis, M.E.; Niyogi, S.; Alvaraez, T.; Kuper, C.; Menon, M.; Haddon, R.C. Effect of rehybridization on the electronic structure of single-walled carbon nanotubes. J. Am. Chem. Soc. 2001, 123, 11292-11293.

78. Ahir, S.V.; Terentjev, E.M.; Lu, S.X.; Panchapakesan, B. Thermal fluctuations, stress relaxation, and actuation in carbon nanotube networks. Phys. Rev. B 2007, doi: 10.1103/PhysRevB.76.165437.

79. Mizuno, K.; Ishii, J.; Kishida, H.; Hayamizu, Y. Yasuda, S.; Futaba, D.N.; Yunura, M.; Hata, K. A black body absorber from vertically aligned single-walled carbon nanotubes. Proc. Natl. Acad. Sci. USA 2009, 106, 6044-6047.

80. Terentjev, E.M.; Warner, M.; Bladon, P. Orientation of nematic elastomers and gels by electric fields. J. Phys. II 1994, 4, 667-676.

81. Urayama. K.; Honda, S.; Takigawa, T. Electrooptical effects with anisotropic deformation in nematic gels. Macromolecules 2005, 38, 3574-3576.

82. Levine, I.; Zvi, R.B.; Winkler, M.; Schmidt, A.M.; Gottlieb, M. Magnetically induced heating in elastomeric nanocomposites-Theory and experiment. Macromol. Symp. 2010, 291-292, 278-286.

83. Winkler, M.; Kaiser, A.; Krause, S.; Finkelmann, H.; Schmidt, A. Magnetoactive Liquid Crystal Elastomers. In Proceedings of the APS March Meeting, New Orleans, LA, USA, 10-14 March 2008.

84. Zadoina, L.; Lonetti, B.; Soulantica, K.; Mingotaud, A.-F.; Respaud, M.; Chaudret, B.; Mauzac, M. Liquid crystalline magnetic materials. J. Mater. Chem. 2009, 19, 8075-8078. 
85. Song, H.M.; Kim, J.C.; Hong, J.H.; Lee, Y.B.; Choi, J.; Lee, J.I.; Kim, W.S.; Kim, J.H.; Hur, N.H. Magnetic and transparent composites by linking liquid crystals to ferrite nanoparticles through covalent networks. Adv. Funct. Mater. 2007, 17, 2070-2076.

86. Garcia-Márquez, A.; Demortière, A.; Heinrich, B.; Guillon, D.; Bégin-Colin, S.; Donnio, B. Iron oxide nanoparticle-containing main-chain liquid crystalline elastomer: Towards soft magnetoactive networks. J. Mater. Chem. 2011, 21, 8994-8996.

87. Kopčanský, P.; Tomašovičová, N.; Koneracká, M.; Závišová, V.; Timko, M.; Džarová, A.; Šprincová, A.; Éber, N.; Fodor-Csorba, K.; Tóth-Katona, T.; Vajda, A.; Jadzyn, J. Structural changes in the 6CHBT liquid crystal doped with spherical, rodlike, and chainlike magnetic particles. Phys. Rev. E 2008, 78, 011702:1-011702:5.

88. Zadoina, L.; Soulantica, K.; Ferrere, S.; Lonetti, B.; Respaud, M.; Mingotaud, A.F.; Falqui, A.; Genovese, A.; Chaudret, B.; Mauzac, M. In situ synthesis of cobalt nanoparticles in functionalized liquid crystalline polymers. J. Mater. Chem. 2011, 21, 6988-6994.

(C) 2012 by the authors; licensee MDPI, Basel, Switzerland. This article is an open access article distributed under the terms and conditions of the Creative Commons Attribution license (http://creativecommons.org/licenses/by/3.0/). 